\title{
Express company's vehicle routing optimization by multiple-dynamic saving algorithm
}

\author{
Junchao Liu ${ }^{1}$, Wei Liu ${ }^{1 *}$, Yuhong $\mathrm{Liu}^{2}$ \\ ${ }^{1}$ College of Transport and Communications, Shanghai Maritime University (China) \\ ${ }^{2}$ Department of Mechanical and Industrial Engineering, University of Toronto (Canada) \\ linj0723@163.com,*Corresponding author weiliu@shmtu.edu.cn, liunuhongsh@gmail.com
}

\begin{abstract}
:
Purpose: According to the disorder in circulation commuting and crossover commuting of SF company which is the China's largest private express delivery service provider, the study uses the Saving Algorithm to make the vehicle routing and resources optimized, on this basis, proposes innovative improvements with Saving Algorithm and then applies it in every distribution center of SF forming a "multi-dynamic" type of Saving Algorithm to ensure both cost savings and timeliness. This method can be generalized for all express company to get the vehicle routing optimized.
\end{abstract}

Design/methodology/approach: As the special transportation requirements of express companies, this study optimizes the vehicle route based on Saving Algorithm, uses multipledynamic Saving Algorithm, and considers the timeliness requirements of the express company to achieve a balance of cost and timeliness.

Findings: The main finding is that a new method proposed which there can be a balance improvement for both cost and timeliness to optimize the vehicle route of express company. Calculation example validates the effectiveness of the model established and solving method.

Practical implications: It is a widespread practice that by setting up model and parameters for the objectives, express company can maintain the balances between cost and timeliness and achieve the optimized vehicle route. 
Originality/value: It proposes innovative improvements, takes SF express company as an example, with Saving Algorithm which can be applied in every distribution center of express company to ensure the balance improvement for both cost and timeliness, and has a great practical significance to the transportation network and path optimization of express companies.

Keywords: express company, saving algorithm, optimization of the vehicle route, multiple-dynamic, limitation, SF company

\section{Introduction}

The express industry in China is developing rapidly in the fierce competition in recent years and has a good prospect in the future. The most important fact of the competitiveness for express company is timeliness and the problems which influence the timeliness, but the research about the ground transportation of express delivery companies are quite few. Scholars have done many studies about applying Saving Algorithm in the VRP, M. Dror and P. Trudeau (Dror \& Trudeau, 1986) proposed a stochastic vehicle routing problem, and introduced an improved saving algorithm, which minimized the anticipated cost and redesigned the vehicle routes. Liu and Liu (2007) studied the vehicle distribution routing problem and proposed an improved saving criteria based on the basic principles of traditional conservation method, which had living examples to demonstrate the improved Saving Algorithm which can reduce the transport distance and cost compared to the typical one. A report summarizes the results of a literature review on dynamic vehicle routing problems, supported by example of real-world applications of such problems, draws a detailed study of the current state of the art in dynamic vehicle routing optimization (Pillac, Guéret \& Medaglia, 2010). A newly survey classifies routing problems from the perspective of information quality and evolution, introduces the notion of degree of dynamism, and presents a comprehensive review of applications and solution methods for dynamic vehicle routing problems (Pillac, Gendreau, Guéret \& Medaglia, 2013). Current and more researches on the logistics vehicle routing optimization are considered from the perspective of cost savings, such as Cao, Zheng, Li, Yang and Lian (2008). They studied the problem of urban pickup and delivery with one way multi load and unload with taking into account the vehicle cost and mileage cost in complicated route net.

These studies above are generally aimed at logistics and distribution enterprises, which have a big difference with express delivery companies. The difference lies in the higher limitation requirement of the express delivery companies which generally follow the prescription of 
"limitation first, considering the cost". Express delivery companies should consider both the transportation cost and the timeliness requirement.

This article considers the special transport requirement of express industry, takes SF express company as an example, optimizing the vehicle routing with Saving Algorithm, and then uses the multiple dynamic Saving Algorithm, with timeliness requirements in mind, to make a balance between the cost and the limitation. It has the significance to the transport network path optimization in express enterprise.

\section{Saving Algorithm}

Saving algorithm is essentially a heuristic algorithm which develops a reasonable path from a scientific point of view, making the entire distribution cost at the lowest level and helps to improve the utilization of vehicles, and often suitable for solving large-scale problems.

\subsection{Model Parameters and Establishment}

$q_{i}(i=1,2, \ldots, n):$ Signify the demand of customer i

$Q_{k}(k=1,2, \ldots K):$ Signify the load capacity of truck $k$

$G$ : Signify the set of all customers, $G=\{1, \ldots n\}$;

$G_{0}=G \cup\{0\},\{0\}$ stands for distribution center

$G_{K}$ : Signify the customer set serviced by truck k

$D_{k}$ : Signify the maximum driving distance of truck $\mathrm{k}$

$C_{i j}$ : Signify all transport costs of the truck from customer $\mathrm{i}$ to customer $\mathrm{j}$.

$n$ : Signify the total number of customers who need service

$t_{i j}$ : Signify the time from customer i to customer $\mathrm{j}$.

$t_{i}$ : Signify the time from DC to customer i

$E T_{i}$ : Signify the earliest allowance start time of task i

$L T_{i}$ : Signify the latest allowance end time of task $\mathrm{i}$

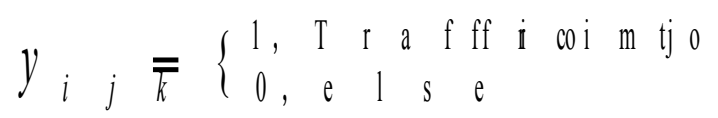




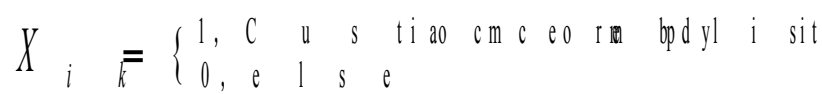

$w_{1}, w_{2}$ : The weight value of each target

$\mu_{i j}$ : The distance from customer i to $\mathrm{j}$

$\delta_{k}$ : The no-load driving cost coefficient of k-truck

According to the above conditions, set the objective function and constraints and establish the mathematical model to optimize the logistics path:

$$
Z=w_{1} m \quad i \sum_{i=1}^{K} \sum_{i=1}^{n} \sum_{j=1}^{n} C_{i} y_{j i}+w_{2} m \quad i \sum_{i=1}^{K} \sum_{i=1}^{n}-\delta_{k} q_{i} x_{k}
$$

s.t.

$$
\begin{aligned}
& \sum_{i=1}^{n} q_{i} x_{i} \leq Q_{k}, \forall k \in K \\
& \sum_{j=1}^{n} y_{i}=x_{j}, \forall j \in G_{0}, k \in K \\
& \sum_{i=1}^{n} y_{i}=_{k} x_{i}, \forall i \in G_{0}, k \in K \\
& \sum_{i=1}^{K} x_{i}=1, i=1,2 \ldots . n \\
& 0 \leq \sum_{i=1}^{n} x_{i} \leq n \\
& \sum_{i, j} \mu_{i} y_{j i j} \leqslant D_{k} \\
& \sum_{i} \sum_{k} x_{i}=n \\
& \sum_{k=1}^{K} x_{i}= \begin{cases}m, & i=0 \\
1, & i=1, \ldots n\end{cases}
\end{aligned}
$$




$$
\begin{gathered}
y_{i j,{ }_{k}} x_{i} \in\{0,1\} \\
\mathrm{i}=0, C_{i j}=C_{0 k}+C_{1 k} \mu_{i j} ; \mathrm{i} \neq 0, C_{i j}=C_{1 k} \mu_{i j}
\end{gathered}
$$

The objective function means the optimal total cost. Constraints: Function (1) constrains the capacity of transport vehicles. Functions (2), and (3) limit the number of vehicle arriving and departing a distribution point is only one. Function (4) means that a distribution point completed only by one truck. Function (5) means that each vehicle with the distribution points cannot exceed the number of all distribution points. Function (6) means that driving distance of each vehicle cannot exceed the maximum travel distance. Function (7) means that all distribution points are loaded. Function (8) ensures that each delivery point is only completed by a truck, but also all distribution points completed by the $\mathrm{m}$ vehicles.

\subsection{To Solve the Saving Algorithm}

Saving Algorithm has used the geometry principle of two sides' length must be longer than the third side. Known that $O$ is the sorting station, it is transported to the sorting station $A$ and $B$ and the distance from $O$ to $A, B$ are $a$ and $b$ respectively. As figures show, it forms two conventional modes of transport:

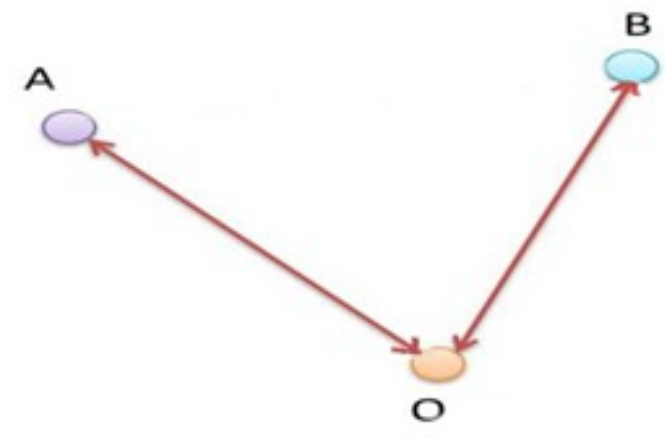

Figure 1. From $O$ to $A, B$ respectively

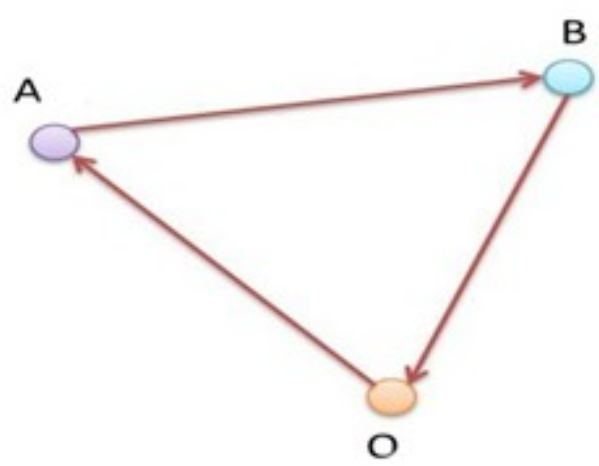

Figure 2. From $O$ to $A, B$ round

In Figure 1 , the delivery distance is $2 \times(a+b)$, in Figure 2, the distribution distance is $a+b$ + c. Obviously, the former need more mileage. Therefore, the core of Saving Algorithm is making two circuits combing into one loop, which minimizes the total transportation distance, until reaching a car loaded limit, and then optimize the next one. 


\section{Apply the Saving Algorithm}

SF company is the China's largest private express delivery service provider which has been providing domestic and international express delivery solutions to a wide-ranging of customers since its establishment in 1993. It has built extensive business units including research and development, logistics, pickup \& delivery, etc. which spans the nation (including Hong Kong, Macau, and Taiwan) and international network covering South Korea, Singapore, Malaysia, Japan, and the United States.

In order to testify the effectiveness of the method, taking China's SF company as an example, we randomly selected 7 points from the East China of SF company. First distribution Hangzhou station is the origin point, assuming that the demand for each sorting station is equivalent to the sorting station handling express number of votes, we get the following data:

\begin{tabular}{|l|r|r|r|r|r|r|}
\hline Customer(i) & Suzhou & Wuxi & Jiaxing & Shaoxing & Ningbo & Wenzhou \\
\hline Freight volume $\left(\mathbf{q}^{\mathbf{i}}\right)$ & 2.8 & 2.3 & 1.8 & 2.4 & 2.3 & 3.3 \\
\hline
\end{tabular}

Table 1. Express Demand for Some Cities (Unit: million votes per day)

At the same time, we use the actual highway mileage between cities, indicating that the distance between cities, thus avoiding errors caused by straight-line distance:

\begin{tabular}{|l|r|r|r|r|r|r|r|}
\cline { 2 - 8 } \multicolumn{1}{c|}{} & \multicolumn{1}{l}{ Hangzhou } & \multicolumn{1}{l}{ Suzhou } & Wuxi & Jiaxing & Shaoxing & Ningbo & Wenzhou \\
\hline Hangzhou & 0 & - & - & - & - & - & - \\
\hline Suzhou & 166 & 0 & - & - & - & - & - \\
\hline Wuxi & 208 & 50.8 & 0 & - & - & - & - \\
\hline Jiaxing & 90.9 & 80.8 & 121 & 0 & - & - & - \\
\hline Shaoxing & 64.2 & 197 & 238 & 123 & 0 & - & - \\
\hline Ningbo & 155 & 230 & 272 & 156 & 117 & 0 & - \\
\hline Wenzhou & 364 & 493 & 532 & 419 & 312 & 269 & 0 \\
\hline
\end{tabular}

Table 2. Road Distance Between Some Cities (Unit: km)

The paper estimates the per kilometer operating costs: $7.1 \mathrm{t}$ truck and $11.2 \mathrm{t}$ truck operating costs are 2.09 Yuan /km, 2.55 Yuan / km.

First, calculate the Distance of saving in same route

\begin{tabular}{|c|c|c|c|c|c|c|}
\hline & Suzhou & & & & & \\
\hline Wuxi & 323.2 & Wuxi & & & & \\
\hline Jiaxing & 176.1 & 177.9 & \begin{tabular}{|l|} 
Jiaxing \\
\end{tabular} & & & \\
\hline Shaoxing & 33.2 & 34.2 & 32.1 & Shaoxing & & \\
\hline Ningbo & 91 & 91 & 89.9 & 102.2 & Ningbo & \\
\hline Wenzhou & 37 & 40 & 35.9 & 116.2 & 250 & Wenzhou \\
\hline
\end{tabular}

Table 3. The Distance of Saving in Same Route 
The second step, to determine transport routes and transport costs of the initial program, now arranged six 7.1t trucks delivery to each sorting station, transport routes and their costs in the table below:

\begin{tabular}{|c|c|c|c|c|}
\hline Transit route & Truck & Distance (km) & $\begin{array}{c}\text { Unit } \\
\text { cost(Yuan/km) }\end{array}$ & $\begin{array}{c}\text { Unit } \\
\text { cost(Yuan/km) }\end{array}$ \\
\hline Hangzhou-Suzhou & $7.1 \mathrm{t}$ & 166 & 2.09 & 2.09 \\
\hline Hangzhou-Wuxi & $7.1 \mathrm{t}$ & 208 & 2.09 & 2.09 \\
\hline Hangzhou-Jiaxing & $7.1 \mathrm{t}$ & 90.9 & 2.09 & 2.09 \\
\hline Hangzhou-Shaoxing & $7.1 \mathrm{t}$ & 64.2 & 2.09 & 2.09 \\
\hline Hangzhou-Ningbo & $7.1 \mathrm{t}$ & 155 & 2.09 & 2.09 \\
\hline Hangzhou-Wenzhou & $7.1 \mathrm{t}$ & 364 & 2.09 & 2.09 \\
\hline Total & & 1048.1 & & $\begin{array}{r}\text { Unit } \\
\text { cost(Yuan/km) }\end{array}$ \\
\hline
\end{tabular}

Table 4. Transport Routes and Transport Costs

According to the basic principles of Saving Algorithm, get the following results of final optimization of the routes:

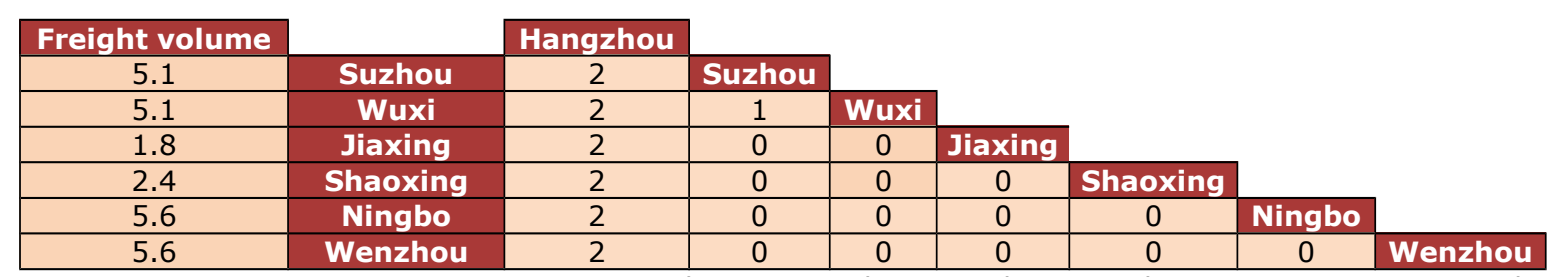

Note: 0 means no connection, 1 means mutual connection between the network, 2 means connecting with distribution center

Table 5. The Final Optimization Results

The modified vehicle scheduling results for:

\begin{tabular}{|l|r|r|r|r|}
\hline Transit route & Truck & Distance(km) & Unit cost(Yuan/km) & Freight(Yuan) \\
\hline Hangzhou-Suzhou-Wuxi & $7.1 \mathrm{t}$ & 216.8 & 2.09 & 453.11 \\
\hline Hangzhou-Jiaxing & $7.1 \mathrm{t}$ & 90.9 & 2.09 & 189.98 \\
\hline Hangzhou-Shaoxing & $7.1 \mathrm{t}$ & 64.2 & 2.09 & 134.18 \\
\hline Hangzhou-Ningbo-Wenzhou & $7.1 \mathrm{t}$ & 424 & 2.09 & 886.16 \\
\hline Total & & 795.9 & & \multicolumn{2}{c}{1663.43} \\
\cline { 3 - 6 }
\end{tabular}

Table 6. Final Scheduling Program and Cost 


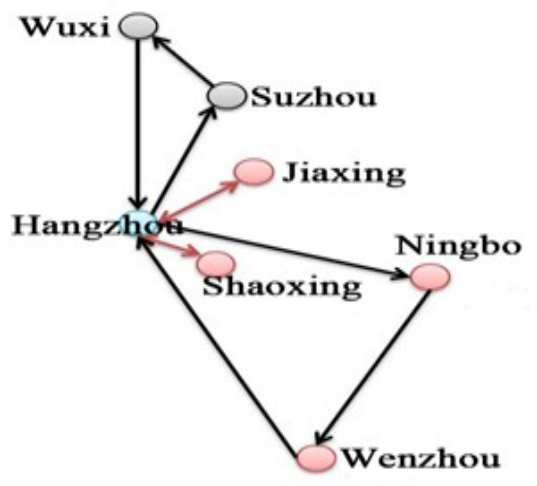

Figure 3(1). The Final Optimization Results

By comparing the initial proposal and the final proposal, the application of the saving algorithm can save about 252.2 kilometer and 527.1yuan. Meanwhile, Saving Algorithm explicitly reveals the relationship among sorting stations, covering every trans-provincial transportation path such as from the level one to the level one and the level one to the level two, and paths from the level two to the level two and the level one to the level two within province, which comparatively fits the present mode of SF.

\section{Multi-dynamic Saving Algorithm}

Single Saving Algorithm is not without flaws. Through its process of calculation, we can know whenever the transport routes form a loop, the total volume of freight in the loop must be less than the maximum load of delivery vehicles, and because of the principle that two sides are longer than the third side, the timeliness will be affected, and the cost and timeliness are unable to unify, that is to say, saving more cost means sacrificing more time.

To compensate for the time loss caused by Saving Algorithm, in this paper, the Saving Algorithm were targeted with feature improvements and provisions.

In eastern China, the approach apply the Saving Algorithm in every distribution center, forming a "multi-dynamic" type of Saving Algorithm to ensure the unity of cost savings and the whole time in SF company. Each sorting station will be the originating point and demand point. When regard second distribution of Ningbo stations as the originating point, we can draw a new independent Saving Algorithm route, as shown: 


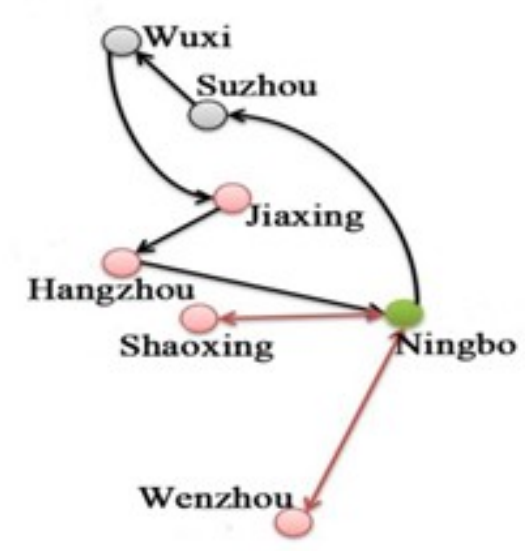

Figure 4. The optimization results of Ningbo origin

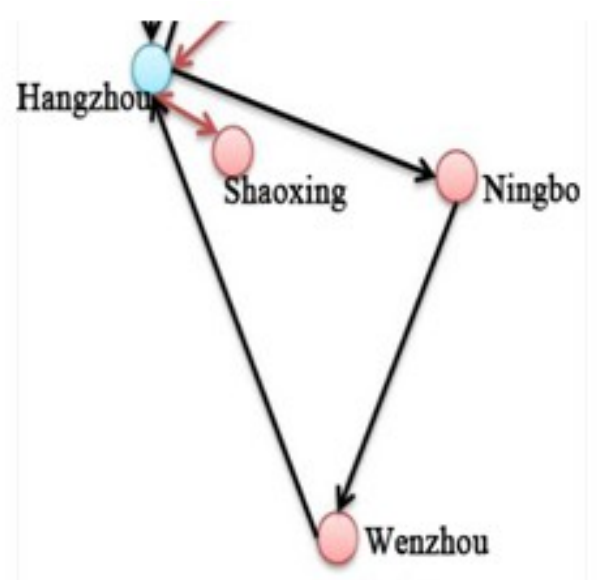

Figure 3(2). The final optimization results

Each individual mileage saving is responsible only for the goods of demand points, and no other waste motion, such as cargo undertaking and exchanging. Such arrangement has the following two advantages:

- it saves the Lay time in sorting points;

- vehicles do not have to wait for the delay in shipment, ensuring the timeliness of the system as a whole.

For example "Hangzhou-Ningbo-Wenzhou" cycle, after the departure of the shipment from Hangzhou, is only responsible for transport to Ningbo and Wenzhou. The truck does not accept the cargo of Hangzhou and Wenzhou at Ningbo.

The saving path of one sorting standing is different in different time, and the route still need to comply with the timeliness requirements of each points. So this paper developed a targeted process. 
The advantage of this set of processes is that the process of saving mileage is always dynamic. When the current batch of express delivery is completed, the vehicle will return to the starting point for the new task. Because each distribution point and cargo number will be different, the route of the vehicle varies.

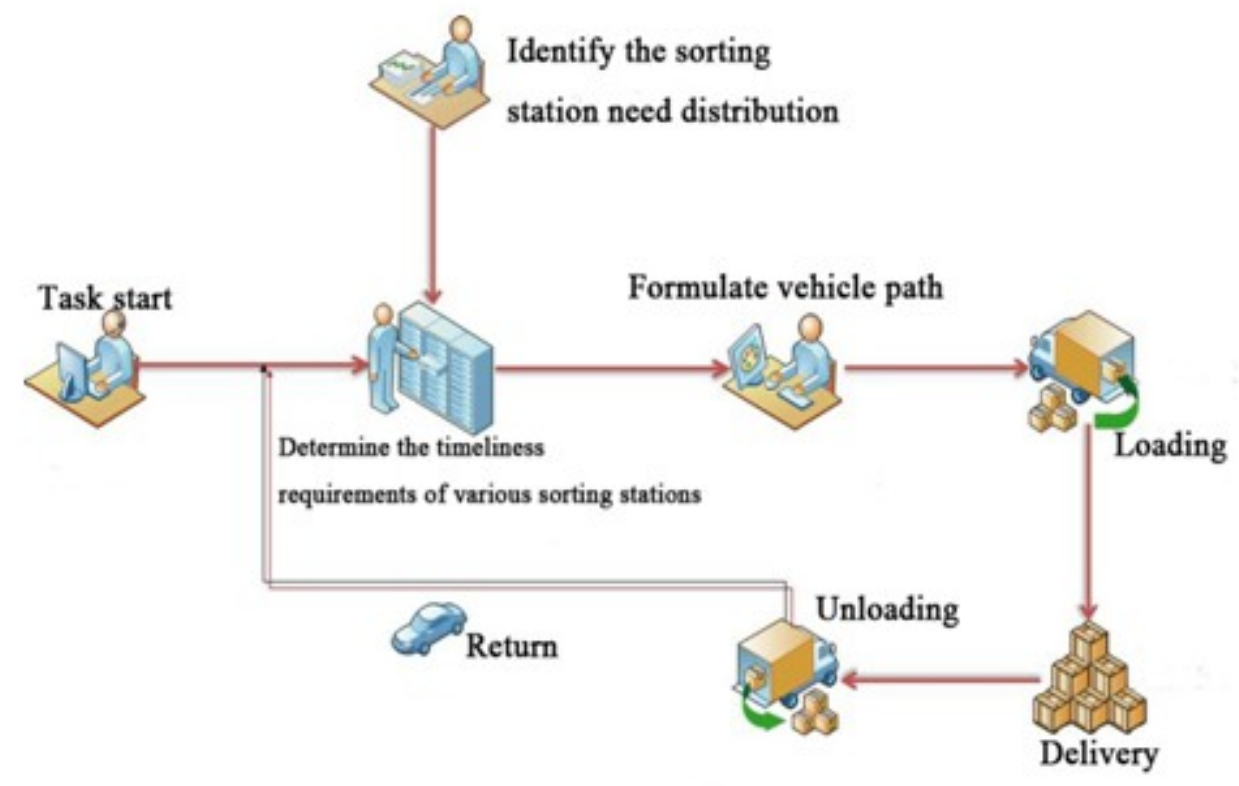

Figure 5. The process of Multiple dynamic Saving Algorithm

\section{Conclusion}

Saving algorithm is essentially a heuristic algorithm, and it helps to improve the utilization of vehicles. It is often suitable for solving large-scale problems. Saving algorithm can develop a reasonable path from a scientific point of view, making the entire distribution cost at the lowest level. Meanwhile, considering the SF Company's overall timeliness, Saving algorithm is applied to various sorting stations, and it develop operational procedures for the SF.

As for saving algorithm, there can be a balance improvement with cost and timeliness. The mileage saving algorithm saves was the trend of diminishing marginal, that is, the more close to the cost-optimal, limitation loss is more. So it can be considered in combination with timeliness, the optimal solution can best meet the cost and timeliness to a certain extent. Although the return path will be no-load, compared to ensure the continuity and timeliness of the overall system, the expense of some sections of the timeliness and cost is acceptable. 


\section{Acknowledgments}

The authors are thankful to the National Natural Science Foundation and the Ministry of Education of China, Shanghai Municipal Education Commission and Science and Technology Commission, and Shanghai Maritime University for providing support for this research (Grant number: 71272219; 11YJA630067; S30601; 11510501800; 20110020, respectively). The authors would also like to express their gratitude to those who have helped them with encouragement, advice, information and material, especially Prof. Tony Hu and Wei Chen who have contributed to the research.

\section{References}

Cao, J.D., Zheng, S.F., Li, B., Yang, Y., \& Lian, X.M. (2008). Optimization of Urban Pickup and Delivery Costs with One Way Multi Load and Unload. Journal of System Simulation, 20(1), 29-32.

Dror, M., \& Trudeau, P. (1986). Stochastic vehicle routing with modified savings algorithm. European Journal of Operational Research, 23(2), 228-235. http://dx.doi.org/10.1016/0377$2217(86) 90242-0$

Liu, W.S., \& Liu, J. (2007). Application of an Improved Economical Method in Delivery Route Optimization. Modern Transportation Technolog, 4(6), 72-74,78.

Pillac, V., Guéret, C., \& Medaglia, A.L. (2010). Dynamic Vehicle Routing Problems: State Of The Art and Prospects. Technical Report, Ecole des Mines de Nantes, France, 1-33.

Pillac, V., Gendreau, M., Guéret, C., \& Medaglia, A.L. (2013). A review of dynamic vehicle routing problems. European Journal of Operational Research, 225(1), 1-11. http://dx.doi.org/10.1016/j.ejor.2012.08.015

Journal of Industrial Engineering and Management, 2014 (www.jiem.org)

Article's contents are provided on a Attribution-Non Commercial 3.0 Creative commons license. Readers are allowed to copy, distribute and communicate article's contents, provided the author's and Journal of Industrial Engineering and Management's names are included. It must not be used for commercial purposes. To see the complete license contents, please visit http://creativecommons.org/licenses/by-nc/3.0/. 